\title{
IMPACTO PSICOSSOCIAL CAUSADO PELA PANDEMIA DA COVID-19 NOS PROFISSIONAIS DE SAÚDE
}

\author{
PSYCHOSOCIAL IMPACT CAUSED BY THE COVID-19 \\ PANDEMIC ON HEALTH PROFESSIONALS
}

\section{IMPACTO PSICOSOCIAL CAUSADO POR LA PANDEMIA COVID-19 EN LOS PROFESIONALES DE LA SALUD}

\author{
Vivian Ranyelle Soares de Almeida ${ }^{1}$ \\ Diana Cardeal do Nascimento ${ }^{2}$ \\ Jenny Caroline Vieira Moura ${ }^{3}$ \\ Juliana Macêdo dos Santos Silva ${ }^{4}$ \\ Denise Rios de Oliveira ${ }^{5}$ \\ Maria Yaná Guimarães Silva Freitas ${ }^{6}$ \\ Carmen Liêta Ressurreição dos Santos
}

Como citar este artigo: Almeida VRS, Nascimento DC, Moura JCV, Silva JMS, Oliveira DR, Freitas MYGS, et al. Impacto psicossocial causado pela pandemia da COVID-19 nos profissionais de saúde. Rev baiana enferm. 2021;35:e37900.

Objetivo: descrever o impacto psicossocial causado pela pandemia do novo coronavírus nos profissionais de saúde. Método: trata-se de revisão narrativa realizada na plataforma da Biblioteca Virtual em Saúde usando os seguintes descritores "Profissionais da saúde" AND "COVID-19", no período de maio a junho de 2020. Resultados: foi desenvolvido um quadro apresentando as características dos seis artigos científicos selecionados, conforme título, autores, ano de publicação, periódico, tipo de estudo e impacto psicossocial. Conclusão: em meio à pandemia os profissionais podem se sentir desamparados, sobrecarregados de atividades e gravemente afetados fisicamente e psicologicamente, tornando-se mais vulneráveis. Faz-se necessário a adoção de medidas protetivas e um plano de ações psicossociais para propiciar melhores condições de trabalho, assistência médica, atendimento psicológico e terapêutico, promovendo qualificações e uso de práticas integrativas e complementares em saúde.

Descritores: Infecções por Coronavírus. Pandemia. Pessoal de Saúde. Impacto Psicossocial.

Objective: to describe the psychosocial impact caused by the pandemic of the new coronavirus on health professionals. Method: this is a narrative review carried out on the Virtual Health Library platform using the following descriptors "Health Professionals" AND "COVID-19", from May to June 2020. Results: a chart was developed showing the characteristics of the six selected scientific articles, according to title, authors, year of publication, journal, type of study and psychosocial impact. Conclusion: in the midst of the pandemic, professionals may feel helpless, overloaded with activities and severely affected physically and psychologically, becoming more vulnerable. There is need to adopt

\footnotetext{
Estudante de Enfermagem. Universidade Estadual de Feira de Santana. Feira de Santana, Bahia, Brasil. ranyalmeida98@hotmail.com. http://orcid.org/0000-00020482-8433.

Estudante de Enfermagem. Universidade Estadual de Feira de Santana. Feira de Santana, Bahia, Brasil. http://orcid.org/0000-000 I-978।-0I54

Estudante de Enfermagem. Universidade Estadual de Feira de Santana. Feira de Santana, Bahia, Brasil. http://orcid.org/0000-0002-444I-3953

Estudante de Enfermagem. Universidade Estadual de Feira de Santana. Feira de Santana, Bahia, Brasil. http://orcid.org/0000-0003-1965-0460

Estudante de Enfermagem. Universidade Estadual de Feira de Santana. Feira de Santana, Bahia, Brasil. http://orcid.org/0000-0003-0470-4577.

6 Enfermeira e Obstetra. Doutora em Saúde Pública. Professora Adjunta da Universidade Estadual de Feira de Santana. Feira de Santana, Bahia, Brasil. http://orcid. org/0000-0002-909|-1566.

Enfermeira e Obstetra. Mestre em Saúde Coletiva. Professora Auxiliar da Universidade Estadual de Feira de Santana. Feira de Santana, Bahia, Brasil. http://orcid. org/0000-000 I-8423-6675.
} 
protective measures and a psychosocial action plan to provide better working conditions, medical care, psychological and therapeutic care, promoting qualifications and the use of integrative and complementary bealth practices.

Descriptors: Coronavirus Infections. Pandemic. Health Personnel. Psychosocial Impact.

Objetivo: describir el impacto psicosocial causado por la pandemia del nuevo coronavirus en los profesionales de la salud. Método: se trata de una revisión narrativa realizada en la plataforma de la Biblioteca Virtual de Salud utilizando los siguientes descriptores "Profesionales de la Salud" AND "COVID-19", de mayo a junio de 2020. Resultados: se desarrolló un cuadro que muestra las características de los seis artículos científicos seleccionados, según el título, los autores, el año de publicación, la revista, el tipo de estudio y el impacto psicosocial. Conclusión: en medio de la pandemia, los profesionales pueden sentirse desolados, sobrecargados de actividades y gravemente afectados física y psicológicamente, volviéndose más vulnerables. Es necesario adoptar medidas de protección y un plan de acción psicosocial para proporcionar mejores condiciones de trabajo, atención médica, atención psicológica y terapéutica, promoción de cualificaciones y el uso de prácticas sanitarias integrativas y complementarias.

Descriptores: Infecciones por Coronavirus. Pandemia. Personal de Salud. Impacto Psicosocial.

\section{Introdução}

A pandemia causada por um vírus denominado SARS-CoV-2 (novo coronavírus) trouxe inúmeros desafios para a população mundial, ocasionando uma situação de alerta e de preocupações sobre as incertezas do futuro próximo, principalmente para os profissionais da saúde que estão na linha de frente contra essa doença.

O vírus é responsável pela COVID-19, uma doença respiratória grave, identificada primeiramente na cidade de Wuhan, na China, que espalhou-se pelo mundo, gerando um grande problema de saúde pública por sua rápida propagação $^{(1)}$. O quantitativo de casos torna-se cada vez mais agravante no Brasil, que é o $2^{\circ}$ país com maior número de casos no mundo inteiro, com 1.841.809 confirmações e 71.540 óbitos notificados até o dia 11 de julho de $2020^{(2)}$.

Embora possa apresentar manifestações clínicas leves, a COVID-19 pode desenvolver sintomas graves, como pneumonia e insuficiência respiratória aguda, podendo levar ao óbito ${ }^{(3)}$. Além de possuir uma elevada transmissibilidade, o vírus pode gerar alta taxa de letalidade para grupos específicos, que possuem determinadas características definidoras, como a idade, a presença de comorbidades e a imunodeficiência ${ }^{(4)}$. $\mathrm{Na}$ busca pela diminuição da quantidade de casos e possível controle da curva epidêmica, tornou-se necessário a adoção de medidas preventivas contra a doença e adaptativas deliberadas pela gestão como: rigorosidade nos hábitos higiênicos, uso de máscara, distanciamento social, restrição de contato, quarentena, bloqueio total (lockdown) e isolamento social ${ }^{(5)}$.

Partindo do pressuposto de controle, além das condições específicas causadas pela patologia, é importante evidenciar que estudos recentes apontaram que essa pandemia tem causado inúmeros reflexos e consequências significativas nas condições psicológicas da população em âmbito mundial, sendo natural que, em situação de ameaça real e iminente, os indivíduos sintam medo ${ }^{(6)}$. Entretanto, os mesmos autores relatam que dependendo do grau de intensidade, esse medo pode gerar ansiedade e mal-estar, aumentando os desafios existentes para o enfrentamento da doença.

Nesse contexto, estima-se que metade da população pode apresentar alguma manifestação psicológica, dependendo de fatores que influenciam a vida cotidiana, como gravidade da pandemia, condição de vulnerabilidade do indivíduo e identificação com o sofrimento de outras pessoas, principalmente os profissionais de saúde e a equipe de enfermagem, que se encontram na linha de frente contra a COVID-19, tornando-se mais vulneráveis também à infecção ${ }^{(7)}$. No Brasil, até o dia 16 de junho de 2020, foram registrados 196 óbitos de enfermeiros, representando $2,44 \%$ da taxa de letalidade para essa categoria ${ }^{(8)}$. 
Portanto, esses profissionais precisam lidar com o enfrentamento direto, o ambiente de adoecimento, o sentimento de luto diante das perdas e o medo pelo risco de adquirir/transmitir a doença, além de ter cuidado redobrado com as medidas protetivas ${ }^{(7)}$. Essas situações podem proporcionar estado de estresse causado pelos impactos psicossociais, que podem ser definidos como as relações entre o meio ambiente e as condições de trabalho, somados às características individuais e familiares dos trabalhadores, podendo interferir de forma negativa na relação indivíduo/ambiente $^{(\vartheta-11)}$.

Os fatores descritos são considerados riscos, podendo revelar traços de personalidade, ocasionar distúrbios emocionais, problemas comportamentais, alterações neuro-hormonais e exacerbar maiores riscos no surgimento de doenças mentais e físicas ${ }^{(10-12)}$. Assim, o termo psicossocial tem sido utilizado para se referir a uma variedade de fatores psicológicos e sociais relacionados com a saúde e a doença no trabalhador ${ }^{(11)}$.

Nessa perspectiva, a escolha do tema deu-se pela necessidade de abordar sobre a saúde profissional em tempos de pandemia, tendo em vista os inúmeros relatos nas mídias de especialistas e dos próprios profissionais sobre as difíceis condições de trabalho que refletem na saúde psicossocial. Além disso, há escassez de produções e estudos que abordem o tema, e se constata uma lacuna do conhecimento pelo fato dessa doença ainda estar sendo estudada e seus impactos em longo prazo sendo mensurados, necessitando de mais conhecimento dos impactos causados em curto prazo.

Acreditamos que este estudo é relevante, pois apresenta um panorama das evidências científicas produzidas recentemente sobre a temática, logo, seus resultados podem contribuir para subsidiar na tomada de decisão, na busca de melhoria nas condições de trabalho, na valorização da equipe de saúde, principalmente dos profissionais de enfermagem, além de evidenciar a necessidade de novos estudos com diferentes abordagens.

Sendo assim, questiona-se: Qual o impacto psicossocial causado pela pandemia da COVID-19 nos profissionais de saúde, encontrado na literatura? O objetivo deste estudo é descrever sobre o impacto psicossocial causado pela pandemia da COVID-19 nos profissionais de saúde.

\section{Método}

Trata-se de revisão narrativa da literatura, que busca demonstrar estudos sobre um determinado tema específico e pouco estudado, incentivando maior alcance e atualização do conhecimento; é julgado como um dos mais benéficos modelos para iniciar um estudo em que se procura afinidades e divergências entre os artigos encon$\operatorname{trados}^{(13-14)}$. Assim, o estudo escolhido viabiliza discorrer sobre o impacto psicossocial causado pela pandemia da COVID-19 nos profissionais da saúde, por meio das publicações que possam dar ênfase ao tema selecionado previamente em banco de dados.

A busca ocorreu nos meses de maio e junho do ano de 2020, na plataforma da Biblioteca Virtual em Saúde (BVS). Inicialmente foi realizada a busca com os descritores "Impacto psicossocial" $A N D$ "Profissionais da saúde" $A N D$ "COVID-19"; posteriormente, utilizou-se "Impacto psicossocial" AND "COVID-19", porém houve escassez de artigos, em número insuficiente para continuidade do estudo. Portanto, foram escolhidos os seguintes descritores "Profissionais da saúde" $A N D$ "COVID-19", sendo evidenciadas inicialmente 430 publicações científicas.

Em seguida, definiu-se como critérios de inclusão, artigos em português, para verificação da produção nacional sobre a temática, publicados no ano de 2020 e que faziam interface com as temáticas impacto psicossocial causado pela COVID-19, profissional de saúde e condições de trabalhos, restando 30 artigos. Após a leitura dos títulos e resumos, foram excluídas 24 produções cientificas por não abordarem a temática delimitada, totalizando 6 artigos para leitura na íntegra.

Para sistematização e análise, os pesquisadores criaram um instrumento para reunir de forma didática as publicações, com as 
seguintes informações: título, autoria, ano de publicação, periódico, tipo de estudo e o impacto psicossocial nos profissionais de saúde ocasionado pela pandemia da COVID-19. Os estudos foram lidos exaustivamente, categorizados e analisados com base no impacto psicossocial.

\section{Resultados}

Com base nos artigos selecionados, foi desenvolvido um quadro com as características desses artigos, conforme título, autores, ano de publicação, periódico, tipo de estudo e impacto psicossocial (Quadro 1).

Quadro 1 - Síntese dos artigos científicos selecionados na plataforma da BVS sobre o impacto psicossocial em profissionais da saúde, conforme título, autores, ano de publicação, periódico, tipo de estudo e impacto psicossocial

\begin{tabular}{|c|c|c|c|c|}
\hline $\mathbf{N}^{\circ}$ & Título & $\begin{array}{c}\text { Autores, Ano, } \\
\text { Periódico }\end{array}$ & $\begin{array}{l}\text { Tipo de } \\
\text { estudo }\end{array}$ & Impacto psicossocial \\
\hline 1 & $\begin{array}{l}\text { Condições de } \\
\text { trabalho e o } \\
\text { impacto na saúde } \\
\text { dos profissionais de } \\
\text { enfermagem frente } \\
\text { a COVID-19 }\end{array}$ & $\begin{array}{l}\text { Miranda FMA, } \\
\text { Santana LL, } \\
\text { Pizzolato AC, Saquis } \\
\text { LMM } \\
\text { (2020) } \\
\text { Cogitare } \\
\text { Enfermagem UFPR }\end{array}$ & Reflexão & $\begin{array}{l}\text { Os profissionais de saúde } \\
\text { apresentam fadiga física, } \\
\text { emocional e mental provocada } \\
\text { pela sobrecarga do processo de } \\
\text { trabalho, estando em posição de } \\
\text { maior vulnerabilidade. }\end{array}$ \\
\hline 2 & $\begin{array}{l}\text { Intervenções em } \\
\text { saúde mental } \\
\text { para profissionais } \\
\text { de saúde frente } \\
\text { a pandemia de } \\
\text { Coronavírus }\end{array}$ & $\begin{array}{l}\text { Saidel MGB, Lima } \\
\text { MHM, Campos } \\
\text { CJG, Loyola CMD, } \\
\text { Esperidião E, Santos } \\
\text { JR } \\
\text { (2020) } \\
\text { Revista Enfermagem } \\
\text { UERJ }\end{array}$ & Reflexão & $\begin{array}{l}\text { Os profissionais da saúde estão } \\
\text { mais vulneráveis a questões } \\
\text { emocionais, estresse pelas } \\
\text { condições de trabalho, medo } \\
\text { de contrair/transmitir o vírus } \\
\text { e incertezas sobre a doença, } \\
\text { causando exaustão da equipe e } \\
\text { esgotamento físico/mental. }\end{array}$ \\
\hline 3 & $\begin{array}{l}\text { Fatores de estresse } \\
\text { nos profissionais } \\
\text { de enfermagem } \\
\text { no combate à } \\
\text { pandemia da } \\
\text { COVID-19: Síntese } \\
\text { de Evidências }\end{array}$ & $\begin{array}{l}\text { Barbosa DJ, Gomes } \\
\text { MP, Souza FBA, } \\
\text { Gomes AMT } \\
\text { (2020) } \\
\text { Comunicação em } \\
\text { Ciências da Saúde }\end{array}$ & $\begin{array}{l}\text { Revisão da } \\
\text { literatura }\end{array}$ & $\begin{array}{l}\text { A equipe de saúde apresentou } \\
\text { níveis elevados de ansiedade, } \\
\text { depressão, medo e estresse } \\
\text { emocional evidenciado pela } \\
\text { sobrecarga do trabalho, falta de } \\
\text { comunicação e treinamento. }\end{array}$ \\
\hline 4 & $\begin{array}{l}\text { Saúde mental dos } \\
\text { profissionais de } \\
\text { enfermagem do } \\
\text { Brasil no contexto } \\
\text { da pandemia } \\
\text { COVID-19: ação do } \\
\text { Conselho Federal } \\
\text { de Enfermagem }\end{array}$ & $\begin{array}{l}\text { Humerez DC, Ohl } \\
\text { RIB, Silva MCN } \\
\text { (2020) } \\
\text { Cogitare } \\
\text { Enfermagem UFPR }\end{array}$ & Reflexão & $\begin{array}{l}\text { Notou-se que os profissionais } \\
\text { da saúde enfrentam incertezas, } \\
\text { anseios e preocupações, causando } \\
\text { problemas na sua saúde } \\
\text { mental e física, apresentando } \\
\text { sentimentos como ansiedade, } \\
\text { estresse, ambivalência, depressão } \\
\text { e exaustão, tornando-os mais } \\
\text { vulneráveis. }\end{array}$ \\
\hline 5 & $\begin{array}{l}\text { Prevenção } \\
\text { relacionada } \\
\text { à exposição } \\
\text { ocupacional do } \\
\text { profissional de } \\
\text { saúde no cenário de } \\
\text { COVID-19 }\end{array}$ & $\begin{array}{l}\text { Gallasch CH, Cunha } \\
\text { ML, Pereira LAS, } \\
\text { Silva-Junior JS } \\
\text { (2020) } \\
\text { Revista Enfermagem } \\
\text { UERJ }\end{array}$ & Reflexão & $\begin{array}{l}\text { Os profissionais da saúde } \\
\text { apresentam alto risco de infecção, } \\
\text { que pode resultar em problemas } \\
\text { emocionais, como estresse, } \\
\text { principalmente pela sobrecarga do } \\
\text { trabalho. }\end{array}$ \\
\hline
\end{tabular}


Quadro 1 - Síntese dos artigos científicos selecionados na plataforma da BVS sobre o impacto psicossocial em profissionais da saúde, conforme título, autores, ano de publicação, periódico, tipo de estudo e impacto psicossocial

\begin{tabular}{|l|l|l|l|l|}
\hline $\mathbf{N}^{\circ}$ & \multicolumn{1}{|c|}{ Título } & \multicolumn{1}{|c|}{$\begin{array}{c}\text { Autores, Ano, } \\
\text { Periódico }\end{array}$} & $\begin{array}{c}\text { Tipo de } \\
\text { estudo }\end{array}$ & \multicolumn{1}{|c|}{ Impacto psicossocial } \\
\hline $\mathbf{6}$ & $\begin{array}{l}\text { A luta dos } \\
\text { profissionais } \\
\text { de saúde no } \\
\text { enfrentamento da } \\
\text { COVID-19 }\end{array}$ & $\begin{array}{l}\text { Medeiros EAS } \\
\text { (2020) } \\
\text { Acta Paulista de } \\
\text { Enfermagem }\end{array}$ & Editorial & $\begin{array}{l}\text { Evidenciou-se que os profissionais } \\
\text { de saúde são mais vulneráveis, } \\
\text { podendo apresentar exaustão física } \\
\text { e mental, risco de infecção, medo } \\
\text { de transmitir a doença e ansiedade. }\end{array}$ \\
\hline
\end{tabular}

Fonte: Elaboração própria.

\section{Discussão}

A COVID-19 ressaltou a urgência de se refletir sobre as alterações psíquicas na população e nos profissionais de saúde, tendo em vista o crescente número de casos e a busca ao atendimento psicológico, o que demonstra vulnerabilidade e impacto negativo na equipe de saúde, com a possibilidade de adoecimento e afastamento do trabalho. Contudo, é comum que durante a pandemia, o destaque seja dado para a descoberta de aspectos epidemiológicos e clínicos de uma nova doença ameaçadora, e menor prioridade para os problemas psíquicos que dela possam advir $^{(15)}$. O aparecimento da pandemia pelo coronavírus evidenciou inúmeras fragilidades dos países afetados no que diz respeito aos aspectos psicológicos da população e, especialmente, dos profissionais de saúde em período de crise ${ }^{(16)}$.

Enquanto milhares de pessoas tendem a ficar em casa para tentar diminuir a transmissão do coronavírus, os profissionais de saúde se colocam em situação contrária a esta, já que necessitam atuar no enfrentamento da pandemia ${ }^{(17)}$. Equipes de saúde que atuam na linha de frente estão mais propensas às alterações emocionais, pois a ausência de comunicação e a falta de treinamento são fatores que predispõem ao risco, elevando a possibilidade do desenvolvimento da COVID-19 e gerando maiores preocupações que podem levar a doenças psicológicas nesses profissionais $^{(15,18)}$.

Além disso, a extensa carga horária de trabalho pode ocasionar um esgotamento físico/mental e a exaustão dos profissionais de saúde ${ }^{(19-20)}$. Estudo realizado com 1.257 profissionais da saúde que estavam cuidando de pacientes infectados com coronavírus evidenciou que 50\% apresentou sintomas depressivos, 45\% ansiedade, 34\% distúrbios do sono e 72\% angústia $^{(21)}$. Em outra pesquisa, os profissionais de saúde relataram: medo devido à grande chance de contrair o vírus e infectar familiares; ansiedade causada pelas notícias divulgadas pelos meios de comunicação; insuficiente quantidade de Equipamentos de Proteção Individual (EPIs); estresse ocasionado pelo elevado e crescente número de pacientes e de óbitos que ocorrem nas unidades de saúde; depressão, devido ao afastamento familiar, óbito dos colegas de trabalho e sentimento de solidão; além da exaustão e/ou esgotamento emocional devido ao elevado volume de trabalho ${ }^{(22)}$.

A utilização de formas de proteção adequadas à situação de pandemia acarreta alterações impactantes nas aquisições e necessidades básicas do cotidiano do profissional de saúde, em decorrência da escassez e/ou racionamento dos insumos e EPIs que lhe são ofertados ${ }^{(15)}$. No entanto, a indispensabilidade desse EPIs e o resguardo físico como regem os protocolos, tanto em nível nacional quanto internacional, garantem maior seguridade no exercício do atendimento em saúde, evitando assim um maior risco de contaminação, mas que pode ocasionar maior exaustão ${ }^{(23)}$.

Embora os profissionais de saúde disponham de conhecimento e preparo no manejo adequado de práticas e técnicas em saúde, a exposição aos agentes patógenos é inevitável, pois estão na 
linha de frente da assistência com cargas elevadas de trabalho, o que contribui para o sofrimento psíquico ${ }^{(16)}$. Associado a esses fatores, agrega-se a necessidade de cuidar e proteger seus pais idosos e/ou filhos pequenos, devido às estratégias de distanciamento social, adotadas como medidas de controle ${ }^{(18)}$. A angústia da proximidade e da distância, essa dicotomia, gera ansiedade e aumenta a possibilidade de desfechos psicológicos.

Assim, a saúde psicológica dos profissionais de saúde pode estar em ameaça e não deve ser menosprezada. Dessa forma, é necessário que os governos enxerguem os trabalhadores, não apenas como prestadores de serviços a serem implementados, mas sim, como seres humanos, aos quais, a segurança e a integridade física/ mental devem ser garantidas ${ }^{(17,20)}$.

Manter a equipe protegida durante a pandemia significa que eles estarão melhor qualificados para desempenharem suas funções. Além da segurança, é necessário que os gestores promovam revezamento de setores; implementação de horários flexíveis para os trabalhadores; redução da carga horária, para evitar maior exposição entre os profissionais, contaminação e afastamento pela doença; liberação de incentivo financeiro e/ou aumento de salários que proporcionem a sobrevivência e condições de assistência digna na vigência de adoecimento; formação de equipe integrada que fortaleça todo o grupo em momentos de instabilidade emocional, e ao mesmo tempo, certificar-se que os profissionais saibam onde e como acessarem os serviços de apoio psicossocial ${ }^{(24)}$.

Outras condutas importantes para redução do nível de estresse são: escuta sensível sobre as angústias diárias que possam ser resolvidas com teleatendimento individualizado; autoconhecimento para detecção de tarefas que possam ocasionar satisfação e liberação da endorfina, conhecida como o hormônio do prazer, que possui efeitos analgésicos, eufóricos e aditivos, consequentemente causando bem-estar ${ }^{(25)}$; estímulo à capacidade em analisar os problemas com percepção positiva evitando o negacionismo e o pessimismo; e uso de Práticas Integrativas e
Complementares em Saúde (PICS), que buscam formas naturais de prevenção de agravos, promoção e recuperação da saúde por meio de tecnologias eficazes e seguras, como Reiki, ventosa, fitoterapia, musicoterapia, uso de medicamentos naturais $^{(26)}$.

\section{Conclusão}

O novo coronavírus trouxe inúmeras mudanças aos profissionais de saúde, podendo trazer impacto negativo na saúde psíquica desses trabalhadores. Em meio a pandemia, os profissionais tendem a se sentirem desamparados, sobrecarregados de atividades e gravemente afetados psicologicamente. Aspectos esses que são intensificados devido às mudanças drásticas na rotina de trabalho, ausência de suporte e meios de enfrentamento, condições de trabalho desfavoráveis com alto índice de exposição ao risco, que proporcionam alta situação de vulnerabilidade.

Dessa forma, é necessário a adoção de medidas protetivas como: assistência médica, redução da carga horária de trabalho, melhor remuneração, realização de rodizio dos profissionais de saúde nos setores críticos e semicríticos, qualificações sobre temas e condutas atuais, educação permanente para sanar dúvidas e proporcionar maior conhecimento, higienização adequada nos ambientes de trabalho, causando maior segurança e consequentemente proteção individual e coletiva.

Ademais, é essencial um plano de ação psicossocial, como atendimento psicológico e terapêutico, escuta sensível sobre o processo de trabalho e angústias diárias, fomentar a autoestima, o autoconhecimento para detecção de tarefas que possam causar bem-estar e o uso de práticas integrativas e complementares.

\section{Colaborações:}

1 - concepção, projeto, análise e interpretação dos dados: Vivian Ranyelle Soares de Almeida, Diana Cardeal do Nascimento, Jenny Caroline Vieira Moura, Juliana Macêdo dos Santos Silva, Denise Rios de Oliveira, Maria 
Yaná Guimarães Silva Freitas e Carmen Liêta Ressurreição dos Santos;

2 - redação do artigo e revisão crítica relevante do conteúdo intelectual: Vivian Ranyelle Soares de Almeida, Diana Cardeal do Nascimento, Jenny Caroline Vieira Moura, Juliana Macêdo dos Santos Silva, Denise Rios de Oliveira, Maria Yaná Guimarães Silva Freitas e Carmen Liêta Ressurreição dos Santos;

3 - aprovação final da versão a ser publicada: Vivian Ranyelle Soares de Almeida, Diana Cardeal do Nascimento, Jenny Caroline Vieira Moura, Juliana Macêdo dos Santos Silva, Denise Rios de Oliveira, Maria Yaná Guimarães Silva Freitas e Carmen Liêta Ressurreição dos Santos.

\section{Referências:}

1. Organização Pan-Americana da Saúde. Folha informativa - COVID-19 (doença causada pelo novo coronavírus) [Internet]. Brasília (DF); 2020 [cited 2020 Jun 6]. Available from: https:// www $\cdot$ paho.org/bra/index.php?option $=\mathrm{com}$ content\&view $=$ article\&id $=6101$ : covid19\&Itemid $=875$

2. Ministério da Saúde. COVID-19 Painel Coronavírus [Internet]. Brasília (DF); 2020 [cited 2020 Jun 10]. Available from: https://covid.saude.gov.br/

3. Farias LABG, Colares MP, Barretoti FKA, Cavalcanti LPG. O papel da atenção primária no combate ao Covid-19: impacto na saúde pública e perspectivas futuras. Rev Bras Med Fam Comunidade [Internet]. 2020 [cited 2020 Jun 7];15(42):1-8. Available from: https:// rbmfc.org.br/rbmfc/article/view/2455/1539

4. Zhu NA, Zhang D, Wang W, Li W, Yang B, Song $\mathrm{J}$, et al. A novel coronavirus from patients with pneumonia in China, 2019. N Engl J Med. 2020;382:727-33. DOI:10.1056/NEJMoa2001017

5. Ministério da Saúde. Secretária de Vigilância em Saúde. Boletim epidemiológico [Internet]. Brasília (DF); 2020 [cited 2020 Jun 7]. Available from: https://www.saude.gov.br/images/pdf/2020/ April/06/2020-04-06-BE7-Boletim-Especial-doCOE-Atualizacao-da-Avaliacao-de-Risco.pdf

6. Silva HGN, Santos LES, Oliveira AKS. Efeitos da pandemia do novo Coronavírus na saúde mental de indivíduos e coletividades. J Nurs Health. 2020;10(esp.):e20104007. DOI: http://dx.doi. org/10.15210/jonah.v10i4.18677
7. Afonso P. O Impacto da Pandemia COVID-19 na Saúde Mental. Acta Med Port. 2020; 33(5):351-8. DOI: https://doi.org/10.20344/amp.13877

8. Conselho Regional de Enfermagem do Espírito Santo. Brasil tem mais de 190 enfermeiros mortos por Covid-19 [Internet]. Vitória (ES); 2020 [cited 2020 Jun 20]. Available from: http://www.coren-es. org.br/brasil-tem-mais-de-190-enfermeirosmortos-por-covid-19_22298.html

9. International Labour Organisation. Psychosocial Factors at Work: Recognition and control [Internet]. Geneva (CH): Occupational Safety and Health Series; 1984 [cited 2020 Jun 7];(56):1-88. Available from: https://www.who.int/occupational_health/ publications/ILO_WHO_1984_report_of_the_ joint_committee.pdf

10. MaissiatGS, Lautert L, Dal PaiD, Tavares JP. Contexto de trabalho, prazer e sofrimento na atenção básica em saúde. Rev Gaúcha Enferm [Internet]. 2015 [cited 2020 Jun 9];36(2):42. Available from: https:// seer.ufrgs.br/RevistaGauchadeEnfermagem/ article/view/51128

11. Trindade LR, Merlo ARC, Silva RM, Beck CLC, Goes NC. Fatores de adoecimento dos trabalhadores da saúde: revisão integrativa. Ciênc Cuid Saúde. 2017;16(4):1-7. DOI: 10.4025/ cienccuidsaude.v16i4.39161

12. Monteiro JK. Sofrimento psíquico de trabalhadores de unidade de terapia intensiva. Rev Psicol: Organ Trab [Internet]. 2012 [cited 2020 Jun 30];12(2):245-50. Available from: http://pepsic.bvsalud.org/scielo.php?script=sci_ arttext\&pid=S198466572012000200009

13. Sousa LMM, Marques-Vieira CMA, Severino SSP, Antunes AV. A Metodologia de Revisão Integrativa da Literatura em Enfermagem. Investigação em Enfermagem [Internet]. 2017 [cited 2020 Jun 9];II(21):17-26. Available from: https://repositorio-cientifico.essatla.pt/bitstream/ 20.500.12253/1311/1/Metodologia $\% 20$ de $\% 20$ Revis\%c3\%a3o\%20Integrativa_RIE21_17-26.pdf

14. Toledo JA, Rodrigues MC. Teoria da mente em adultos: uma revisão narrativa da literatura. Bol - Acad Paul Psicol [Internet]. 2017 [cited 2020 Jun 9];37(92):139-56. Available from: http://pepsic.bvsalud.org/scielo.php?script $=$ sci_ arttext\&pid=S1415-711X2017000100011\&lng=pt\& nrm $=$ iso

15. Barbosa DJ, Gomes MP, Souza FBA, Gomes AMT. Fatores de estresse nos profissionais de 
enfermagem no combate à pandemia da COVID19: Síntese de Evidências. Com Ciências Saúde [Internet]. 2020 [cited 2020 Jul 30];31(Suppl 1):31-47. Available from: http://www.escs.edu.br/revistaccs/ index.php/comunicacaoemcienciasdasaude/ article/view/651/291

16. Saidel MGB, Lima MHM, Campos CJG, Loyola CMD, Esperidião E, Santos JR. Intervenções em saúde mental para profissionais de saúde frente a pandemia de Coronavírus. Rev enferm UERJ. 2020;28:e49923. DOI: http:// dx.doi.org/10.12957/reuerj.2020.49923

17. Remuzzi A, Remuzzi G. COVID-19 and Italy: what next? The Lancet [Internet]. 2020;395:1225-8. DOI: https://doi.org/10.1016/ S0140-6736(20)30627-9

18. Medeiros EAS. A luta dos profissionais de saúde no enfrentamento da COVID-19. Acta paul enferm. 2020;33:e-EDT20200003. DOI: http://dx.doi. org/10.37689/acta-ape/2020edt0003

19. Miranda FMA, Santana LL, Pizzolato AC, Saquis LMM. Condições de trabalho e o impacto na saúde dos profissionais de enfermagem frente a Covid-19. Cogitare enferm. 2020;25:e72702. DOI: http://dx.doi.org/10.5380/ce.v25i0.72702

20. Xiang Y, Jin Y, Wang Y, Zhang Q, Zhang L, Cheung T. Tribute to health workers in China: A group of respectable population during the outbreak of the COVID-19. Int $\mathrm{J}$ Biol Sci. 202;16(10):1739-40. DOI: 10.7150/ijbs. 45135

21. Lai J, Ma S, Wang Y, Cai Z, Hu J, Wei N, et al. Factors Associated With Mental Health Outcomes Among Health Care Workers Exposed to Coronavirus Disease 2019. JAMA Netw Open. 2020;3(3):e203976. DOI:10.1001/ jamanetworkopen.2020.3976
22. Humerez DC, Ohl RIB, Silva MCN. Saúde mental dos profissionais de enfermagem do Brasil no contexto da pandemia covid-19: ação do Conselho Federal de Enfermagem. Cogitare enferm. 2020;25:e74115. DOI: http://dx.doi.org/10.5380/ ce.v25i0.74115

23. Gallasch CH, Cunha ML, Pereira LAS, Silva-Junior JS. Prevenção relacionada à exposição ocupacional: COVID-19. Rev enferm UERJ. 2020;28:e495961. DOI: http://x.doi.org/10.12957/ reuerj. 2020.49596

24. Organização Pan-Americana da Saúde. Considerações psicossociais e de saúde mental durante o surto de COVID-19 [Internet]. Washington D.C. (EUA); 2020 [cited 2020 Jul 5]. Available from: https://iris.paho.org/bitstream/ handle/10665.2/51996/OPASBRACOVID1920040_ por.pdf?sequence $=1 \&$ isAllowed $=y$

25. Werneck FZ, Bara Filho MG, Ribeiro LCS. Mecanismos de Melhoria do Humor após o Exercício: Revisitando a Hipótese das Endorfinas. $\mathrm{R}$ bras Ci e Mov [Internet]. 2005 [cited 2020 Aug 6];13(2):135-44. Available from: https:// portalrevistas.ucb.br/index.php/RBCM/article/ viewFile/634/645

26. Brasil. Ministério da Saúde. Glossário temático: práticas integrativas e complementares em saúde [Internet]. Brasília (DF); 2018 [cited 2020 Aug 6]. Available from: http://portalarquivos2.saude.gov. br/images/pdf/2018/marco/12/glossario-tematico. pdf

Recebido: 14 de julho de 2020

Aprovado: 14 de agosto de 2020

Publicado: 26 de novembro de 2020

A Revista Baiana de Enfermagem utiliza a Licença Creative Commons - Atribuição-NãoComercial 4.0 Internacional. https://creativecommons.org/licenses/by-nc/4.0/

Este artigo é de acesso aberto distribuído sob os termos da Licença Creative Commons (CC BY-NC). Esta licença permite que outros remixem, adaptem e criem a partir do seu trabalho para fins não comerciais. Embora os novos trabalhos tenham de lhe atribuir o devido crédito e não possam ser usados para fins comerciais, os usuários não têm de licenciar esses trabalhos derivados sob os mesmos termos. 